\title{
USE OF RAPID-DEPLOYMENT EXTRACORPOREAL MEMBRANE OXYGENATION FOR THE RESUSCITATION OF PEDIATRIC PATIENTS WITH HEART DISEASE AFTER CARDIAC ARREST
}

Brian W. Duncan, MD Andra E. Ibrahim, $\mathrm{MD}^{\mathrm{b}}$ Viktor Hraska, MD ${ }^{\text {a }}$

Pedro J. del Nido, MD $^{\mathrm{a}}$

Peter C. Laussen, $\mathrm{MD}^{\mathrm{c}}$

David L. Wessel, MD

John E. Mayer, Jr., MD ${ }^{\mathrm{a}}$

Lynne K. Bower, CCP, RRT ${ }^{\mathrm{d}}$

Richard A. Jonas, MD ${ }^{\mathrm{a}}$
Introduction: We have recently used extracorporeal membrane oxygenation as a means of rapidly resuscitating pediatric patients with heart disease after cardiopulmonary arrest, in whom conventional resuscitation measures have failed. Methods: We developed a fully portable extracorporeal membrane oxygenation circuit that is maintained vacuum and carbon dioxide-primed at all times. When needed, the circuit is crystalloid-primed and can be ready for use within 15 minutes. Since February 1996, we have used this rapid-deployment circuit to resuscitate 11 pediatric patients in full cardiopulmonary arrest. Results: The median age of the 11 patients was 120 days ( 2 days to 4.6 years). Nine patients had a cardiac arrest after cardiac surgery. One patient had a cardiac arrest during cardiac catheterization and one patient had a cardiac arrest before cardiac surgery. Median duration of cardiopulmonary resuscitation was 55 minutes (range 20 to 103 minutes), with no difference in the duration of cardiopulmonary resuscitation between survivors and nonsurvivors. Ten of 11 patients $(91 \%)$ were weaned from extracorporeal membrane oxygenation and seven (64\%) survived to hospital discharge. Six patients are long-term survivors, five of whom are in New York Heart Association class I; one survivor is in class II. Seven patients resuscitated with extracorporeal membrane oxygenation before the use of this rapid-deployment circuit had a median duration of cardiopulmonary resuscitation of 90 minutes, with two $(28.6 \%)$ survivors. Conclusions: The use of rapid-deployment extracorporeal membrane oxygenation results in shorter resuscitation times and improved survival in pediatric patients with heart disease after cardiopulmonary arrest. (J Thorac Cardiovasc Surg 1998;116:305-11)
C ardiac arrest may complicate the course of any child with cardiac disease, particularly in the postoperative period after heart surgery for congenital heart disease. These children represent the most critically ill subset of an already challenging patient population. Conventional basic and advanced cardiopulmonary resuscitation (CPR) in adults and children has often yielded dismal results. Mechanical circulatory support has

From the Departments of Cardiac Surgery, ${ }^{\text {a }}$ Anesthesia, ${ }^{\mathrm{b}}$ Cardiology, ${ }^{\mathrm{c}}$ and Respiratory Care, ${ }^{\mathrm{d}}$ Children's Hospital, Boston, Mass.

Received for publication Dec. 29, 1997; revisions requested Feb. 9, 1998; revisions received March 31, 1998; accepted for publication March 31, 1998.

Address for reprints: Richard A. Jonas, MD, Department of Cardiac Surgery, Children's Hospital, 300 Longwood Ave., Boston, MA 02114.

Copyright (C) 1998 by Mosby, Inc.

$0022-5223 / 98 \$ 5.00+0 \quad \mathbf{1 2 / 1 / 9 0 6 6 3}$ been used in adult patients with cardiac arrest in a number of settings but without significant improvement in outcome..$^{1-3}$

The use of extracorporeal membrane oxygenation (ECMO) to resuscitate children having a cardiac arrest after cardiac surgery has demonstrated better results than in many other patient groups. ${ }^{4,5}$ In our institution the subset of pediatric patients with cardiac disease requiring ECMO for cardiac arrest demonstrated survivals comparable with those for all pediatric patients with heart disease requiring ECMO, for all other indications. ${ }^{6}$ These results suggest that cardiac arrest in pediatric patients with cardiac disease may be due to reversible conditions that can be successfully treated with ECMO support. This report details our approach using a modified ECMO circuit and an organized team to facilitate the rapid resuscitation of pediatric patients with heart disease after cardiac arrest. 
Table I. Patient characteristics

\begin{tabular}{ll}
\hline Sex & \\
$\mathrm{M}$ & 8 \\
$\mathrm{~F}$ & 3 \\
Age & 120 days $(2$ days-4.6 yr $)$ \\
Weight $(\mathrm{kg})$ & $4.9(2.5-15.6)$ \\
Time after operation $(\mathrm{hr})(n=9)$ & $10(2-192)$ \\
Duration of ECMO $(\mathrm{hr})$ & $65(2-210)$ \\
Days chest open & $6.5(0-27)$ \\
Days intubated & $13.5(4-78)$ \\
Total hospital days & $29.5(10-65)$ \\
\hline
\end{tabular}

Median values are listed with ranges in parentheses.

\section{Methods}

Patient population and statistical analysis. A retrospective chart review was undertaken of 11 pediatric patients with heart disease who were treated with rapidresuscitation ECMO beginning in February 1996. All children were in full cardiopulmonary arrest, receiving $\mathrm{CPR}$ at the time of ECMO cannulation. The charts from seven patients who were resuscitated with ECMO after having a cardiac arrest before the use of this rapiddeployment circuit were reviewed for comparison of hospital course and outcome. These historical control patients were resuscitated with the standard ECMO circuit before February 1996 and were included in a previous analysis of the hospital course of pediatric patients with cardiac disease undergoing mechanical circulatory support. ${ }^{6}$ Follow-up data were obtained by telephone interview with parents and pediatricians of these patients after written informed consent was obtained from the parents. All data procurement and patient follow-up were carried out after institutional approval according to guidelines established by the Committee for Human Experimentation of Children's Hospital.

Data were analyzed with a statistical program (JMP Software, SAS Institute, Inc., Cary, N.C.). Comparison of the duration of CPR between survivors and nonsurvivors of the rapid-resuscitation group and between the rapidresuscitation group overall and historical controls was made with the Wilcoxon two-group test. Comparisons between survivals for the rapid-resuscitation group and historical controls was performed with Pearson's $\chi^{2}$ test. Confidence limits (CL) listed for proportions (see Results) are equivalent to one standard error.

Components of the rapid-resuscitation ECMO circuit. Our standard ECMO setup has been previously described. ${ }^{6,7}$ The rapid-resuscitation circuit is modified to allow the institution of support within 15 minutes of notification of its need. Maintained in the intensive care unit on a cart with a portable, battery power supply, the circuit is completely mobile and can be wheeled to any location in the hospital. The circuit is maintained with a vacuum and carbon dioxide-primed $0.8 \mathrm{~m}^{2}$ membrane oxygenator (Avecor Cardiovascular, Inc., Plymouth, Minn.) in line. This oxygenator is capable of supporting flows of $1.2 \mathrm{~L} / \mathrm{min}$ suitable for children up to $10 \mathrm{~kg}$. For larger children up to $25 \mathrm{~kg}$, a $1.5 \mathrm{~m}^{2}$ membrane is spliced into the circuit $(1.8 \mathrm{~L} / \mathrm{min}$ maximum flow rate).
When needed for resuscitation of patients who have postoperative cardiac arrest, direct aortic and atrial cannulation through the chest usually provides the most expeditious means of instituting support while allowing the performance of effective, open CPR. In patients who have had cardiac arrest in settings other than the postoperative period, femoral or neck cannulation is performed at the discretion of the surgeon. The rapid-resuscitation circuit is Normosol solution (Abbott Laboratories, Abbott Park, Ill.) primed with $50 \mathrm{ml}$ of $5 \%$ albumin and debubbled at the bedside. Normosol solution has been substituted for the standard saline prime in the rapidresuscitation circuit to minimize electrolyte disturbances with the institution of support. The circuit is connected to the cannulas and ECMO support is initiated.

Addition of blood components. When deployment of the rapid-resuscitation ECMO circuit is requested, the blood bank is also notified to procure blood products for addition to the circuit. If blood products are not available when cannulation is complete, support is initiated with the crystalloid primed circuit and then $200 \mathrm{ml}$ of packed red blood cells, $100 \mathrm{ml}$ of fresh frozen plasma, and 2 units of cryoprecipitate are added to the circuit when available. For those patients who have support instituted with a crystalloid primed circuit, blood is usually available for addition to the circuit within 15 to 60 minutes of the institution of support. Blood is then added to the circuit and crystalloid volume is removed by means of a one-forone syringe exchange transfusion. We use the Amicon ultrafiltration system (W. R. Grace and Co., Beverly, Mass.) to further remove crystalloid volume as blood products are added, once hemodynamics on support have stabilized.

Rapid-resuscitation ECMO team. In our institution, ECMO is managed by the Respiratory Care Department, which provides personnel in the hospital at all times for the institution of urgent ECMO support. One in-house respiratory therapist carries the "priming beeper." If a pediatric patient with heart disease has a cardiac arrest and does not respond to standard resuscitative measures after 10 minutes, a cardiologist or cardiac surgeon initiates a call for rapid-resuscitation ECMO. Respiratory care personnel transport the circuit to the patient's bedside and initiate priming. On-call members of cardiac surgery, cardiology, and cardiac nursing are also notified that emergency ECMO is required. Cardiac surgeons perform all ECMO cannulations for patients with heart disease in our institution. Once support is instituted, members of the Respiratory Care Department continue to manage the circuit.

\section{Results}

Demographics and setting of support. Demographic data for the patients treated with rapidresuscitation ECMO are listed in Table I. Seven of the 11 patients were less than 1 year of age and three of the 11 were less than 30 days of age. Nine of the 11 patients had support instituted in the postoperative period (Table II). Patient 2, with pulmonary atresia and an intact ventricular septum, had sudden 
Table II. Diagnoses, procedures, and outcomes

\begin{tabular}{|c|c|c|c|c|c|c|}
\hline Patient & Setting & $\begin{array}{l}\text { Age } \\
\text { (days) }\end{array}$ & $\begin{array}{l}\text { Weight } \\
(\mathrm{kg})\end{array}$ & Diagnosis & Procedure & Outcome \\
\hline 1 & Postop. & 207 & 4.9 & DORV-VSD & Baffle of VSD to aorta & Survived \\
\hline 2 & Preop. & 5 & 2.9 & PA-IVS & TAP, right MBTS & Survived \\
\hline 3 & Postop. & 2 & 4.5 & HLHS & Norwood procedure & Died \\
\hline 4 & Postop. & 12 & 2.9 & Heterotaxy, PA & Left MBTS & Survived \\
\hline 5 & Postop. & 567 & 10.5 & Mitral regurgitation & Mitral valve repair & Died \\
\hline 6 & Cath. lab. & 1667 & 15.6 & TOF-PA & Coiling of aortopulmonary collaterals & Survived \\
\hline 7 & Postop. & 109 & 4.9 & Transitional AV canal & $\begin{array}{l}\text { Patch closure of ASD, VSD; } \\
\text { mitral valve repair }\end{array}$ & Survived \\
\hline 8 & Postop. & 631 & 10.5 & Corrected TGA, PA & $\begin{array}{l}\text { IVC-TV baffle; VSD-aorta baffle; } \\
\text { RV-PA conduit }\end{array}$ & Survived \\
\hline 9 & Postop. & 1120 & 12.0 & Rejection & Status post cardiac transplantation & Late death \\
\hline 10 & Postop. & 58 & 2.5 & TOF & TOF repair (VSD closure; TAP) & Died \\
\hline 11 & Postop. & 39 & 3.3 & Truncus arteriosus & $\begin{array}{l}\text { Truncus arteriosus repair } \\
\text { (VSD closure, RV-PA conduit) }\end{array}$ & Died \\
\hline
\end{tabular}

$D O R V$, Double-outlet right ventricle; $V S D$, ventricular septal defect; $P A$, pulmonary atresia; $I V S$, intact ventricular septum; $T A P$, transannular patch; $M B T S$, modified Blalock-Taussig shunt; HLHS, hypoplastic left heart syndrome; TOF, tetralogy of Fallot; $A V$, atrioventricular; $A S D$, atrial septal defect; $T G A$, transposition of the great arteries; $I V C$, inferior vena cava; $T V$, tricuspid valve; $R V-P A$, right ventricle-pulmonary artery.

ventricular fibrillation during an otherwise stable preoperative course. After stabilization and diagnostic cardiac catheterization on ECMO, the patient was transported to the operating room where conversion to standard cardiopulmonary bypass for surgical repair was performed. Because of postoperative hypoxia, ECMO was continued by means of chest cannulation. The patient was subsequently weaned and survived. Patient 6, with tetralogy of Fallot and pulmonary atresia, had support instituted in the cardiac catheterization laboratory after cardiac arrest caused by hypoxia while aortopulmonary collaterals were being coiled. This patient was resuscitated with ECMO and eventually discharged, with definitive repair being performed during a subsequent hospitalization. The diagnoses of the seven historical control patients included ventricular septal defect with aortic coarctation, tricuspid atresia, transposition of the great arteries, transposition of the great arteries with ventricular septal defect, severe mitral regurgitation after repair of a transitional atrioventricular canal, total anomalous pulmonary venous return, and complete atrioventricular canal.

Nonsurvivors. Patient 3, who had hypoplastic left heart syndrome, had ventricular failure and cardiac arrest after a Norwood procedure (Table II). This patient was successfully resuscitated with ECMO and was eventually weaned from support. However, the patient subsequently died of multisystem organ failure after cardiac transplantation. Patient 5 had a cardiac arrest caused by progressive left ventricular failure after mitral valve repair. After resuscitation with ECMO the patient was unable to be weaned from support and underwent cardiac transplantation. A mycotic aneurysm of the aortic anastomosis of the cardiac allograft subsequently developed and caused the patient's death. Patient 10, an infant with multiple congenital anomalies, had sudden cardiac arrest the night after repair of tetralogy of Fallot, presumably caused by arrhythmia. During cannulation, open cardiac compressions were discontinued for substantial lengths of time. This resulted in severe hypoxic damage to all organ systems, and ECMO was electively discontinued the following day. This was the only patient in this series who could not be successfully weaned from ECMO. Patient 11 had a sudden cardiac arrest after truncus arteriosus repair, possibly caused by a pulmonary hypertensive crisis. ECMO was instituted and eventually weaned successfully; however, within 24 hours of discontinuing ECMO supersystemic pulmonary artery pressures developed and failed to respond to conventional measures. ECMO was again instituted via neck cannulation for an additional 6 days, after which overwhelming mediastinal sepsis developed, resulting in the patient's death. Patient 9 represents the only late death in this series. This patient was born with hypoplastic left heart syndrome and underwent cardiac transplantation for progressive ventricular dysfunction at 3 years of age after a Norwood procedure and a subsequent bidirectional Glenn shunt. This patient had a cardiac arrest during an episode of severe rejection and was successfully weaned from ECMO after treatment of the rejection episode. The patient was discharged from the hospital with mild hypoxic encephalopathy, 


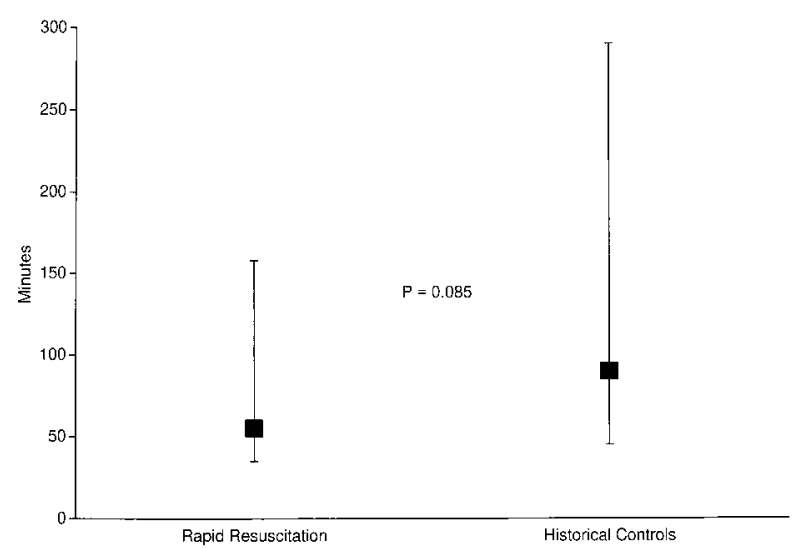

Fig. 1. Duration of CPR for patients resuscitated with rapid-resuscitation ECMO since February $1996(n=11)$ compared with historical controls resuscitated with standard ECMO before $1996(n=7)$.

but disseminated lymphoma eventually developed, resulting in death.

Sites of cannulation. Seven patients were cannulated through the chest in the immediate postoperative period. Patient 8 had a cardiac arrest the night of operation after a complex reconstruction for corrected transposition of the great arteries with pulmonary atresia and was cannulated through the chest. This patient subsequently received a left ventricular assist device after a successful resuscitation and a brief period (2 hours) of ECMO support. Patient 11 was cannulated through the chest in the immediate postoperative period and was successfully weaned from ECMO but was recannulated via the neck on postoperative day 8 as discussed above. Patients 4 and 9 had a cardiac arrest 10 and 8 days after cardiac surgery, respectively, and were cannulated through the neck. Patient 2 was cannulated preoperatively through the neck and was recannulated through the chest for postoperative support as detailed above. Patient 6 had ECMO initiated in the cardiac catheterization laboratory via sheaths in the femoral vessels while neck cannulation was being performed and was subsequently converted to neck cannulation.

Complications. Cardiovascular complications (Table III) developed in two patients who required cardiac transplantation owing to lack of return of native cardiac function on ECMO and in two patients with significant ventricular ectopy. The three pulmonary complications included pulmonary infarction, pulmonary hemorrhage, and pneumothorax in one patient each. The gastrointestinal com-
Table III. Complications

\begin{tabular}{lc}
\hline \multicolumn{1}{c}{ Complication } & No. of Patients \\
\hline Cardiovascular & 4 \\
Pulmonary & 3 \\
Gastrointestinal & 3 \\
Renal failure & 2 \\
Neurologic & 4 \\
Hemorrhage & 2 \\
Any infection & 5 \\
Positive blood cultures & 3 \\
Mediastinitis & 3 \\
Pneumonia & 2 \\
Mechanical & 1 \\
\hline
\end{tabular}

plications included upper gastrointestinal tract hemorrhage resulting from gastritis, nutritional failure necessitating gastrostomy tube placement, and unexplained chronic elevation of enzyme levels on liver function tests. Two patients had renal failure necessitating dialysis. One of these patients had recovery of renal function by the time of hospital discharge, but in the other patient renal function did not recover before death. Neurologic complications occurred in four patients: two had mild hypoxic encephalopathy, one had choreoathetosis, and one patient had seizures. The single mechanical complication was due to dislodgment of the aortic cannula 30 minutes after support was initiated. The patient was quickly recannulated successfully.

Outcome. Fig. 1 compares the median duration of $\mathrm{CPR}$ in patients receiving rapid-resuscitation ECMO with seven historical control patients resuscitated with a standard ECMO circuit before 1996. The median duration of CPR was 55 minutes (range 20 to 103 minutes) for the rapid-resuscitation group and 90 minutes (range 45 to 200 minutes) for the historical controls. The duration of CPR was over 30 minutes less with the rapid-resuscitation approach $(p=0.085)$. The durations of CPR for survivors (58 minutes, range 25 to 103 minutes) and nonsurvivors (51 minutes, range 20 to 83 minutes) in the rapidresuscitation group were not significantly different $(p=0.63)$. Fig. 2 compares the outcomes between the rapid-resuscitation group and the historical controls. A single patient in the rapid-resuscitation group could not be weaned from ECMO. The hospital survival in the rapid-resuscitation group (7/11 survivors; $64 \%$; CL $45 \%$ to $81 \%$ ) represents a substantial improvement over that of the historical control group (2/7 survivors; 29\%; CL $10 \%$ to 55\%; $p=0.15)$. Fig. 3 demonstrates the New York Heart Association functional status in the six long-term 


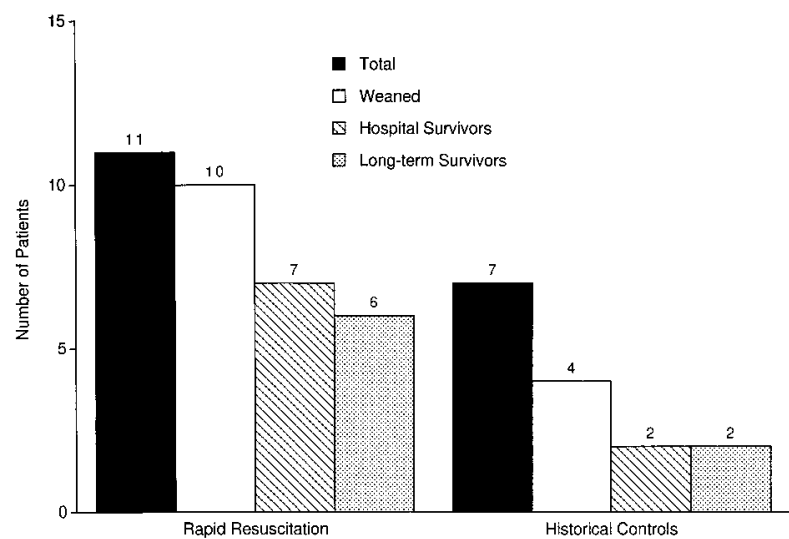

Fig. 2. Outcomes for the rapid-resuscitation ECMO group and historical controls. Total patients undergoing ECMO resuscitation, patients successfully weaned from ECMO, in-hospital survivors, and long-term survivors are demonstrated for both groups.

survivors after rapid-resuscitation ECMO. All but one survivor is in New York Heart Association class I with one patient in class II. One of the six long-term survivors has mild neurologic impairment, and one child has moderate impairment (Fig. 4).

Prime composition and outcome. Data regarding the composition of the ECMO prime at the time of institution of support is available for eight of the 11 patients. Six of these eight patients had support instituted with crystalloid solution and two had support instituted with a blood-primed circuit. Of the six patients who received ECMO support with a circuit initially primed with crystalloid, five (83\%) were successfully weaned from support and four $(67 \%)$ survived to hospital discharge. Both patients supported with a blood-primed ECMO circuit were successfully weaned from support and one of these patients $(50 \%)$ survived to hospital discharge.

\section{Discussion}

Mechanical circulatory support for the resuscitation of patients who suffer a cardiac arrest has gained increasingly widespread use in a number of settings. ${ }^{1-3,8,9}$ The application of mechanical circulatory support for this indication has arisen from the dismal outcome of conventional resuscitative measures in these patients. ${ }^{10,11}$ Despite initial enthusiasm for ECMO in the setting of cardiac arrest in adults, substantial mortality persists in most reports. ${ }^{2}$ However, previous reports describing ECMO to support pediatric patients with heart

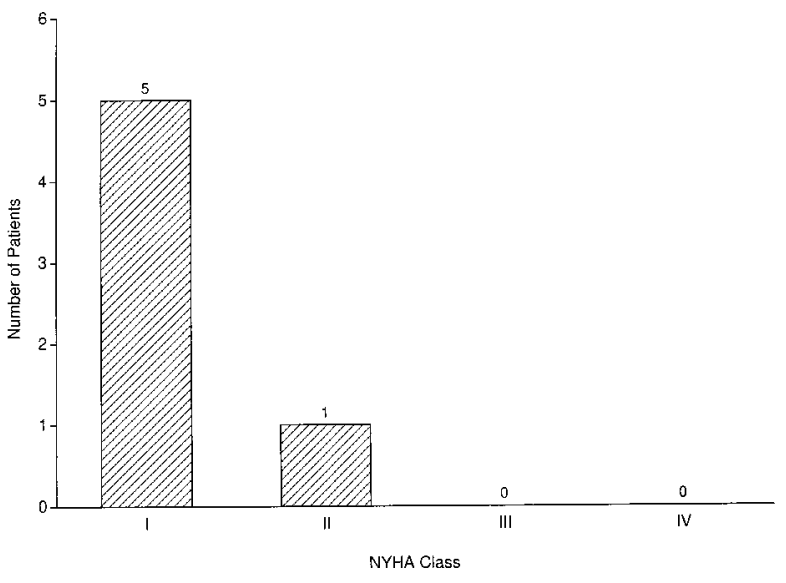

Fig. 3. New York Heart Association (NYHA) classification of six long-term survivors after rapid-resuscitation ECMO.

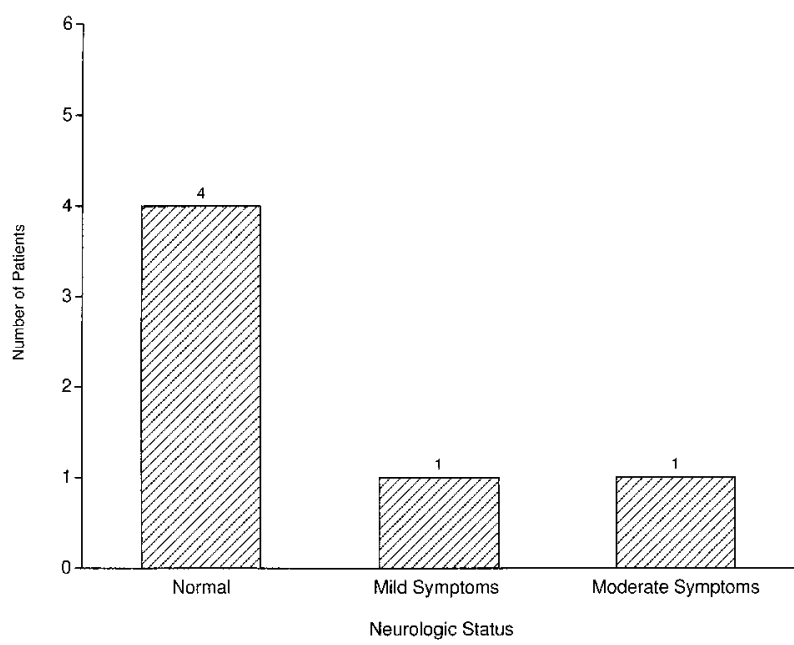

Fig. 4. Neurologic status of six long-term survivors after rapid-resuscitation ECMO.

disease after a cardiac arrest suggest their outcomes may be better, justifying an aggressive approach in their resuscitation. ${ }^{4,5} \mathrm{~A}$ review of the in-hospital course of pediatric patients with heart disease treated with mechanical circulatory support in our institution demonstrated that the survival for patients requiring support for cardiac arrest was the same as for all pediatric patients with heart disease requiring ECMO support (40\% survival). ${ }^{6}$

The reasons for better outcomes in pediatric patients with heart disease may relate to witnessed arrests in an intensive care unit setting, the use of effective open CPR in the postoperative period, and 
a greater degree of core cooling during cardiac arrest in children than in adults. In addition, cardiac arrest in these children is often due to a transient arrhythmia or other reversible cause such as pulmonary hypertensive crisis. The impact of core cooling and effective CPR is supported by the data in the present study, in which 10 of 11 patients were successfully weaned from ECMO with excellent end-organ function. Only a single patient had evidence of significant hypoperfusion during resuscitation requiring termination of support because of advanced end-organ failure.

We believe that the most important factor in the successful resuscitation of these patients is the prompt establishment of an adequate cardiac output. This rapid-resuscitation approach begins with a prompt decision to institute ECMO support in patients who have not responded to routine resuscitative measures after cardiac arrest. We currently initiate a call for rapid-resuscitation ECMO for our patients who have a cardiac arrest if CPR is unsuccessful after 10 minutes. Other important components of this approach include using a modified ECMO circuit to facilitate rapidly instituting support and creating an organized team to minimize response times. In our prior experience we found that routine priming of the ECMO circuit led to delays in the institution of support. A source of significant delay was consistently incurred while waiting for blood products and the subsequent blood priming step. If blood is not available, we currently institute support with a crystalloid-primed circuit to establish adequate hemodynamics as quickly as possible. The majority of the patients in this series had support instituted with a crystalloidprimed circuit without apparent detriment to endorgan function. Blood is added to the circuit when it becomes available, which is usually within $30 \mathrm{~min}$ utes of the institution of ECMO. We have not used O negative or type-specific blood; however, if excessive delays in receiving cross-matched blood occur, the use of non-crossmatched blood products could be considered. Ultrafiltration can be performed as blood is added to remove crystalloid volume from the circuit when hemodynamics have stabilized. The organization of a rapid-response team centered around in-house personnel who are available at all times to perform circuit priming is also of vital importance. As circuit priming is initiated, cardiology, cardiac surgery, and cardiac nursing members of the rapid-response team are notified so that cannulation and direction of resuscitative efforts may proceed simultaneously. With this approach, we presently can institute support within 30 to 45 minutes of cardiac arrest in most instances.

Once support is instituted, this patient population requires meticulous management. Temporary ventricular dysfunction is often profound. We aggressively monitor for left ventricular distention by transesophageal echocardiography and left-sided filling pressures. We incorporate a left atrial vent into the circuit or perform balloon atrial septostomy if left ventricular distention occurs. Balloon atrial septostomy may be performed in the cardiac catheterization laboratory or at the bedside under echocardiographic guidance. ${ }^{12}$ Four patients in this series required left atrial venting and three patients had an unrestrictive interatrial communication to limit left ventricular distention. If left ventricular distension is avoided, many of these patients can be expected to require relatively brief periods of ECMO support, as further evidence of the reversible nature of the cause of cardiac arrest in the majority of these cases. Early return of ventricular function (within 48 to 72 hours of institution of support) was seen in all patients who were subsequently weaned from ECMO and did not require heart transplantation. This is a consistent finding from our previous analysis. ${ }^{6}$

Shunted, single ventricle physiology does not represent a contraindication to ECMO resuscitation after cardiac arrest. Partially occluding the shunt during ECMO is often useful to provide balanced pulmonary and systemic circulations. In this series, three patients with shunted, single ventricle physiology (pulmonary atresia, intact ventricular septum, complex heterotaxy with single ventricle, and hypoplastic left heart syndrome) were successfully resuscitated and supported. Two of these patients are long-term survivors; the third patient was successfully weaned from support but died after cardiac transplantation.

The complications and deaths in this series are an indication of the gravity of the clinical condition of these patients. Bleeding complications are common in patients undergoing postoperative mechanical circulatory support, and in our previous analysis excessive blood loss was a risk factor for in-hospital death. ${ }^{6}$ Mediastinal sepsis was also a common source of morbidity and mortality in this patient population. Two of the four deaths were the result of mediastinal infections that were undoubtedly due to the fact that the majority of these children were cannulated through the chest. The development of 
percutaneous systems for femoral cannulation for children may eliminate lengthy periods during which the chest is open, leading to a decrease in the incidence of mediastinitis in these patients.

Neurologic complications were less common than might be predicted for this group of patients, attesting to the importance of effective CPR and prompt ECMO institution. We further attempt to lessen the impact of central nervous system ischemia occurring during resuscitation by aggressive, controlled hypothermia. ${ }^{13}$ This begins at the time of cardiac arrest by placing ice around the patient's head during cannulation while CPR is performed. We then maintain patient temperature at $34^{\circ} \mathrm{C}$ or less during the first 24 hours of support and avoid temperature elevations above $37^{\circ} \mathrm{C}$ at any time during the first few days of support. Long-term neurologic follow-up will be necessary to determine the ultimate functional status of these children.

\section{Summary}

Pediatric patients with heart disease appear to represent a favorable subset of patients who have cardiac arrest in the hospital. Apparently reversible causes of cardiac arrest in these patients may be quickly diagnosed and treated during brief periods of ECMO support. The most important factor leading to a successful outcome in these children is the prompt institution of ECMO to provide a normal cardiac output. We have developed a portable ECMO circuit that can be ready for the institution of support within 15 minutes of cardiac arrest. Using this system for resuscitation has resulted in the survival of seven of $11(64 \%)$ pediatric patients with heart disease who have had a cardiac arrest.

We thank Kimberlee Gauvreau, ScD, for her invaluable assistance in the statistical analysis of these data.

\section{REFERENCES}

1. Phillips SJ, Zeff RH, Kongtahworn C, Skinner JR, Toon RS, Grignon A, et al. Percutaneous cardiopulmonary bypass: application and indication for use. Ann Thorac Surg 1989;47:121-3.

2. Dembitsky WP, Moreno-Cabral R, Adamson RM, Daily PO. Emergency resuscitation using portable extracorporeal membrane oxygenation. Ann Thorac Surg 1993;55:304-9.

3. Grambow DW, Deeb M, Pavlides GS, Margulis A, O'Neill WW, Bates ER. Emergent percutaneous cardiopulmonary bypass in patients having cardiovascular collapse in the cardiac catheterization laboratory. Am J Cardiol 1994;73:872-5.

4. del Nido PJ, Dalton HJ, Thompson AE, Siewers RD. Extracorporeal membrane oxygenator rescue in children during cardiac arrest after cardiac surgery. Circulation 1992; 86(Suppl):II300-4.

5. Dalton HJ, Siewers RD, Fuhrman BP, del Nido PJ, Thompson AE, Shaver MG, et al. Extracorporeal membrane oxygenation for cardiac rescue in children with severe myocardial dysfunction. Crit Care Med 1993;21:1020-8.

6. Duncan BW, Hraska V, Jonas RA, Blume ED, del Nido PJ, Wessel DL, et al. Mechanical circulatory support for pediatric cardiac patients. Circulation 1996;94(Suppl):I173.

7. Wilson JM, Bower LK, Fackler JC, Beals DA, Bergus BO, Kevy SV. Aminocaproic acid decreases the incidence of intracranial hemorrhage and other hemorrhagic complications of ECMO. J Pediatr Surg 1993;28:536-41.

8. Laub GW, Banaszak D, Kupferschmid J, Magovern GJ, Young JC. Percutaneous cardiopulmonary bypass for treatment of hypothermic circulatory collapse. Ann Thorac Surg 1989;47:608-11.

9. Goldman AP, Kerr SJ, Butt W, Marsh MJ, Murdoch IA, Paul T, et al. Extracorporeal support for intractable cardiorespiratory failure due to meningococcal disease. Lancet 1997;349:466-9.

10. Ballew KA, Philbrick JT, Caven DE, Schorling JB. Predictors of survival following in-hospital cardiopulmonary resuscitation. Arch Intern Med 1994;154:2426-32.

11. Zaritsky A. Outcome of pediatric cardiopulmonary resuscitation. Crit Care Med 1993;21(Suppl):S325-7.

12. O'Connor TA, Downing GJ, Ewing LL, Gowdamarajan R. Echocardiographically guided balloon atrial septostomy during extracorporeal membrane oxygenation (ECMO). Pediatr Cardiol 1993;14:167-8.

13. Lanier WL. Cerebral metabolic rate and hypothermia: their relationship with ischemic neurologic injury. J Neurosurg Anesthesiol 1995;7:216-21. 